\title{
Preface to the special issue on user modeling to support groups, communities and collaboration
}

\author{
Elena Gaudioso · Amy Soller • Julita Vassileva
}

(C) Springer Science+Business Media B.V. 2006

Technology should support and enhance our ability to work, learn, and make decisions. These activities naturally involve collaboration and communication, yet the personal computers we use to carry them out are traditionally individualized. This special issue addresses the design and extension of adaptive individual user technology to support complex collaborative processes involving information sharing, communication, and collaborative group work.

Recent integration efforts have successfully combined collaborative and communication services with multi-user tools to create environments that enable individuals to communicate and share information. Examples of such environments include computer-supported collaborative workspaces, distributed learning management systems, online communities of interest, and peer-to-peer systems. While these environments enable collaboration, they provide no guarantee that timely productive collaboration occurs and proceeds effectively. Such assurance requires more substantial effort in understanding how the collaboration process is shaped by the individuals' characteristics, behaviors, and relationships, and how to support their dynamic interaction. Models of groups, collaboration, and communities collect and structure the

E. Gaudioso $(\varangle)$

Department of Artificial Intelligence,

School of Computer Science, Spanish University for Distance Education (UNED),

Juan del Rosal-16, 28040 Madrid, Spain

e-mail: elena@dia.uned.es

\author{
A. Soller \\ Institute for Defense Analyses, \\ 4850 Mark Center Drive, Alexandria, VA 22311, USA \\ e-mail: asoller@ida.org \\ J. Vassileva \\ University of Saskatchewan, Department of Computer Science, \\ 176 Thorvaldson Bldg., 110 Science Place, Saskatoon, Saskatchewan, \\ S7N 5C9, Canada \\ e-mail: jiv@cs.usask.ca
}


rich information describing interactions between users. This special issue considers a variety of these models, and discusses the key challenges they face.

Group and community models may combine or extend individual models with multi-user features, or they may take on new designs that model group characteristics in innovative ways. For example, the article by Harrer, McLaren, Walker, Bollen and Sewall discusses the way in which traditional individual tutoring model tracing methods can be extended to analyze group activities. The authors' model tracing approach is an example-based approach similar to decision tree learning that characterizes group behavior in terms of learning productivity. In their paradigm, successful groups may be characterized by the way in which they alternate between chatting and taking problem solving actions, move between different subtasks, or distribute their work among group members.

Suebnukarn and Haddawy describe a general domain-independent framework for modeling individual and group problem solving, and its application to a collaborative intelligent tutoring system for medical problem-based learning. Bayesian Networks are used to represent student and group models that overlay a model of clinical reasoning and problem-based learning. While the structure of the networks is derived from research papers and textbooks on clinical reasoning and problem-based learning, the conditional probabilities are automatically learned from data collected during a problem-based learning study.

In Suebnukarn and Haddawy's approach, the group model is generated as a consensus among the individual models by overlaying the problem solving paths most likely to be followed by the individual members. The tutoring module then generates tutorial hints that guide the group problem solving activity, for example by focusing the group discussion or referring to an expert in the group. The authors describe the evaluation of their system, and show that their model predicts individual student actions and the group reasoning path with high accuracy (compared to human tutors).

Group modeling may involve consideration of dynamic social factors that are not present in individual models, such as interactions and relationships between users. Interaction data may be obtained from unstructured communication channels such as discussion forums, text chat, instant messaging, or voice-over-IP. Because analyzing unstructured voice or chat data is computationally challenging, if not manually exhausting, some systems solve this problem by structuring the collaboration environment. For example, Introne and Alterman discuss how coordinating representations structure the collaborative environment and assist users in exchanging and interpreting the information they share. The authors show how their Bayesian approach exploits the rich coordination information between users to improve coordination and performance. They have demonstrated the effectiveness of their approach in an existing groupware system and have shown how it can be extended with an adaptive plan-generation component.

Multi-user environments introduce complex relational social elements such as emotion, motivation, and satisfaction. Masthoff and Gatt explain that the ability of a system to model and predict users' affective states such as satisfaction will impact its ability to adapt to a group's needs. In the context of a system that recommends sequences of items to groups of users, they describe how an individual's satisfaction may impact the satisfaction of other group members by analyzing factors such as emotional contagion and conformity. They also discuss the issue of privacy in aggregating individual 
preferences to make group-based recommendations that might divulge personal (and perhaps embarrassing) partialities.

Cheng and Vassileva address the problem of how to motivate participation in online communities and collaborative systems. In their approach, individuals are rewarded when they take actions that are desirable for the community. The authors propose an adaptive rewards approach that utilizes a group (community) model and individual models of the users. The group model plays a prescriptive role, by describing the dynamics of the desirable types of contributions and establishing the rewards accordingly. The individual models represent each user's contribution in terms of quantity and quality (reputation), and are used to adjust rewards in order to encourage users to contribute. Cheng and Vassileva describe how this mechanism was evaluated in a system for sharing bookmarks, and show that it is effective.

Determining how to structure and coordinate group membership poses a significant challenge in designing and developing technology for supporting collaborative work. Organizations or tasks may place specific requirements on group membership such as individuals' physical, cognitive, or social skills, experience, or availability. Read, Barros, Bárcena, and Pancorbo describe an adaptive group formation algorithm in the context of a system for group language learning. They show how a system that maintains and draws upon both individual and group models may be able to construct groups that incorporate a variety of linguistic skills and social contexts and thereby produce a more realistic language learning environment.

Alfonseca, Carro, Martín, Ortigosa, and Paredes investigate the effect of learning styles on the outcome of collaborative pairs. The Felder-Silverman model is used to represent the learning styles of 166 students. The results of a study where the students are paired by their own choice to solve two theoretical computer science assignments substantiate previous results in the area of education in which heterogeneous teams do no worse (usually better) than homogeneous teams. The authors explain how they apply these results to create rules for team-formation in a system that supports collaboration.

The authors contributing to this special issue have developed novel approaches to address challenging questions in modeling group and community collaboration. We hope that you find their research ideas and approaches interesting, informative, and refreshing. This issue highlights some exciting developing areas of research to watch in the future including modeling affective states, social relationships, and group decision-making, analysis of interaction, coordination in cooperative multi-agent systems, multi-user plan recognition, and multiple perspective social networking.

\section{Authors' Vitae}

Elena Gaudioso is an Associate Professor of Artificial Intelligence and Computer Science at UNED. She received her Ph.D. degree in Artificial Intelligence and Computer Science from UNED in 2003. Her research interests lie in the areas of artificial intelligence in education, machine learning and user modeling. Currently she is working in applying data mining techniques to support tutoring in web-based educational systems and in virtual learning communities. She has authored over 20 papers and has been member of a number of scientific program committees.

Amy Soller is a Research Staff Member in the Science and Technology Division at the Institute for Defense Analyses. She holds a B.S. in Electrical Engineering, and a Ph.D. in Intelligent Systems from the University of Pittsburgh. Her research involves applying intelligent methods to analyze and assess collaborative problem solving, and providing advice on national security issues concerning perfor- 
mance support, information sharing, and distributed collaboration technology. She was previously a Senior Artificial Intelligence Engineer at the MITRE Corporation, and a Project Manager at the Institute for Research in Science and Technology, Italy, designing intelligent technology solutions for facilitating information sharing, knowledge management, community awareness, and organizational learning. She has published over 40 peer reviewed articles and sits on a number of editorial and scientific advisory boards.

Julita Vassileva is an Associate Professor of Computer Science at the University of Saskatchewan. She received her Ph.D. degree in Mathematics and Computer Science from the University of Sofia, Bulgaria in 1992. Dr. Vassileva has worked in the areas of artificial intelligence in education, adaptive hypertext and hypermedia and multi-agent systems. More recently, her research has focused on ways to encourage participation in online communities, personal information management as well as recommendation systems using trust and reputation mechanisms. She has authored over hundred technical papers and has co-edited two books. Dr. Vassileva was program co-chair of the 8th international conference on User Modelling in Sonthofen, Germany, 2001. 